\title{
EDITORIAI
}

\section{DESARROLLO DEL MASTER Y DOCTORADO OFICIAL DE ENFERMERÍA EN ESPAÑA.}

La monografía Desarrollo del Master y Doctorado oficial de enfermería en España, se presentó el pasado 27 de Enero en la Universitat Jaume I. Se trata de un documento historiográfico escrito por las personas que generaron y dinamizaron la formación avanzada de la enfermería actual en nuestro país durante el periodo 1998-2006.

Esta narrativa analiza la trayectoria realizada desde las Escuelas de Enfermería del país que apostaron por situar a la formación de la enfermería española en el nivel académico más elevado posible. Esta motivación fue el motor de cambio para aprovechar el momento en el que fuese una realidad el crecimiento de la ciencia enfermera. Estos hechos históricos han permitido que actualmente muchas enfermeras tengan el grado de doctor y que muchas otras estén realizando sus trabajos de investigación con la posibilidad de generar conocimiento dentro de la disciplina Enfermera.

La repercusión del desarrollo disciplinar puede no detectarse por el gran colectivo enfermero asistencial en un corto plazo, ya que el ámbito asistencial absorbe el mayor volumen de trabajo de enfermería y en ocasiones tiene dificultades por acceder al rendimiento real que genera un desarrollo disciplinar. Sin embargo a través de un ejemplo práctico se puede detectar la repercusión del mencionado desarrollo:

"Quien tiene más posibilidades de realizar investigación son las enfermeras docentes....si estas realizan investigación clínica aplicada en equipo con enfermeras asistenciales...hablaran todas el mismo lenguaje y coordinaran proyectos. Si ese lenguaje es comprensible y los resultados de la investigación se trasladan a la práctica asistencial.....las enfermeras asistenciales verán mejorar su trabajo con el apoyo metodológico de las enfermeras doctoras...y verán mejorar las actividades asistenciales basadas en resultados de investigaciones aplicadas que habrán elaborado conjuntamente y empezara a ser normal que una enfermera doctora y una no doctora trabajen en equipo y apliquen sus hallazgos sobre los pacientes a los que atienden. Se mejorara la aplicación de procedimientos....los resultados de efectos adversos de la hospitalización y la atención domiciliaria entre otras cosas. En definitiva....la 
sociedad gana a través del trabajo de las enfermeras".

Esta aportación es la que se logra con iniciativas similares a las que recoge la mencionada monografía.

Consta de dos partes bien diferenciadas. La primera parte, con un total de cinco capítulos, es el relato histórico en sí mismo donde los protagonistas son reales y las referencias se avalan con actas de reuniones y convocatorias. Allí encontramos las debilidades internas, fortalezas, amenazas y oportunidades de una profesión en crecimiento.

En la segunda parte se relata por capítulos, la percepción de las escuelas participantes en el proceso de cambio, con un orden de información homogéneo, datos contrastables e impresiones de las directoras.

La obra concluye con una visión de futuro dirigida a las próximas generaciones de enfermería, que sirva de mensaje de que la enfermería no es propiedad de nadie. La ciencia de la Enfermería requiere de las aportaciones científicas de sus profesionales para aportar mejoras en el cuidado de la salud de la población.

Finalmente, hay que destacar que esta monografía se ha podido publicar, gracias a la dotación económica que obtuvo la Dra. Zabalegui del Premio competitivo a la Iniciativa Docente al proyecto "Master y Doctorado en Ciencias de la Enfermería" del Ilustre Colegio Oficial de Enfermería de Barcelona, 2006. Además, también ha contribuido el departamento de publicaciones de la Universitat Jaume I de Castellón, que sin haber implantado los estudios, apostó por ellos.

* Loreto Maciá Soler, es responsable de la implantación de estudios de Enfermería Grado, Master y Doctorado de la Universitat Jaume I de Castellón.

Adelaida Zabalegui Yárnoz, es Directora de Enfermería del Hospital Clinic de Barcelona. 